\title{
TREINAMENTO AUDITIVO PARA TRANSTORNO DO PROCESSAMENTO AUDITIVO: UMA PROPOSTA DE INTERVENÇÃO TERAPÊUTICA
}

\author{
Auditory training for auditory processing disorder: \\ a proposal for therapeutic intervention
}

Alessandra Giannella Samelli (1), Fabíola Ferrer Del Nero Mecca ${ }^{(2)}$

\section{RESUMO}

Objetivo: verificar a eficácia de um programa informal de treinamento auditivo específico para transtornos do Processamento Auditivo, em um grupo de pacientes com esta alteração, por meio da comparação de pré e pós-testes. Métodos: participaram deste estudo 10 indivíduos de ambos os sexos, da faixa etária entre sete e 20 anos. Todos realizaram avaliação audiológica completa e do processamento auditivo (testes: Fala com Ruído, Sttagered Spondaic Word - SSW, Dicótico de Dígitos, Padrão de Frequência). Após 10 sessões individuais de treinamento auditivo, nas quais foram trabalhadas diretamente as habilidades auditivas alteradas, a avaliação do processamento auditivo foi refeita. Resultados: as porcentagens médias de acertos nas situações pré e pós-treinamento auditivo demonstraram diferenças estatisticamente significantes em todos os testes realizados. Conclusão: o programa de treinamento auditivo informal empregado mostrou-se eficaz em um grupo de pacientes com transtorno do processamento auditivo, uma vez que determinou diferença estatisticamente significante entre o desempenho pré e pós-testes na avaliação do processamento auditivo, indicando melhora das habilidades auditivas alteradas.

DESCRITORES: Percepção Auditiva; Transtornos da Audição; Estimulação Acústica; Plasticidade Neuronal

\section{INTRODUÇÃO}

O processamento auditivo refere-se à eficiência e à efetividade pelas quais o sistema nervoso central utiliza a informação auditiva. Uma falha neste mecanismo neural dá origem a um transtorno do processamento auditivo. Por sua vez, este transtorno pode levar ou estar associado a dificuldades de linguagem, aprendizagem e funções comunicativas $^{1-3}$.

(1) Fonoaudióloga; Professora Doutora do Departamento de Fisioterapia, Fonoaudiologia e Terapia Ocupacional da Faculdade de Medicina da Universidade de São Paulo, FMUSP, São Paulo, SP; Doutora em Ciências pela Faculdade de Medicina da Universidade de São Paulo.

(2) Fonoaudióloga; Professora do Curso de Fonoaudiologia da Universidade Guarulhos, UNG, Guarulhos, SP; Doutora em Educação pela Universidade de São Paulo.

Conflito de interesses: inexistente
Em casos de transtornos do processamento auditivo diagnosticados, em virtude do impacto destas alterações na audição, na comunicação e no sucesso acadêmico, é necessária uma intervenção rápida, por meio de programas baseados no treinamento auditivo e na melhora do sinal acústico, além do emprego de estratégias de linguagem, cognitivas e metacognitivas, os quais promoverão a plasticidade e a reorganização cortical ${ }^{1-3}$.

A neuroplasticidade auditiva pode ser definida como a alteração das células nervosas para melhor responderem às influências ambientais, as quais são críticas para a memória e aprendizado. A plasticidade pode ser observada por meio de mudanças comportamentais e eletrofisiológicas ${ }^{2-7}$. Muitas investigações demonstraram estas mudanças, após treinamento auditivo ${ }^{2,7-18}$.

Existem diversos programas de treinamento auditivo voltados para indivíduos com transtornos de processamento auditivo, de linguagem oral ou 
escrita, bem como de aprendizagem. Em revisão feita sobre 0 assunto ${ }^{9}$ muitos destes programas foram descritos, sendo que alguns são mais ecléticos e trabalham diversas habilidades auditivas, enquanto outros se fixam numa determinada forma de treino. No que se refere ainda aos programas de treinamento auditivo, alguns autores ${ }^{2}$ afirmaram que geralmente incorporam uma programação de treinamento intensivo e estímulos específicos para atingir um determinado alvo, usando técnicas adaptadas para rapidamente melhorar o processamento auditivo. Outra autora ${ }^{4}$ acrescentou que existem dois tipos básicos de treinamento auditivo: o formal, no qual um equipamento eletroacústico e/ ou programas de computadores são utilizados, e o informal, no qual o treinamento é realizado em casa com os pais ou na escola com os professores, em virtude da não disponibilidade de equipamentos sofisticados.

O objetivo do presente estudo foi verificar a eficácia de um programa informal de treinamento auditivo específico para transtornos do Processamento Auditivo, em um grupo de pacientes com esta alteração, por meio da comparação de pré e pós-testes.

\section{MÉTODOS}

Este estudo foi do tipo transversal prospectivo. Participaram 10 indivíduos que foram atendidos na Clínica de Fonoaudiologia da Universidade GuaruIhos (UnG). O grupo foi composto por oito participantes do sexo masculino e dois do feminino, sendo que: três tinham sete anos, dois tinham oito anos, dois tinham nove anos, um participante tinha 11 , um 15 anos e um 20 anos. Os participantes serão citados por número de 1 a 10, distribuídos por faixa etária de forma crescente.

Todos os participantes foram autorizados por seus pais para participar do estudo, pela assinatura do Termo de Consentimento Livre e Esclarecido.

Os critérios de seleção da amostra foram: avaliação audiológica básica dentro dos limites da normalidade; sem queixas e história atuais de afecções do sistema auditivo; apresentar a avaliação do Processamento Auditivo alterada.

Inicialmente, todos os pacientes passaram por avaliação audiológica básica (imitanciometria, audiometria tonal limiar e logoaudiometria) para que qualquer alteração de sistema auditivo periférico fosse descartada.

Em seguida, realizaram avaliação comportamental do processamento auditivo aplicado por meio do audiômetro Interacoustic AC 40 acoplado a um $C D$ player da marca Sony. Foram aplicados dois testes monóticos e dois dicóticos ${ }^{19,20}$.
Foram aplicados os seguintes testes: Fala com Ruído, para avaliar principalmente a habilidade de fechamento auditivo; o Sttagered Spondaic Word (SSW), para avaliar as habilidades de análise e síntese, memória, figura-fundo para sons linguísticos, associação som-símbolo; e o Dicótico de Dígitos (etapa de integração), que avalia figura-fundo para sons linguísticos e integração binaural ${ }^{19}$. Foi aplicado também o Teste de Padrão de Frequência (TPF), o qual avalia principalmente a habilidade de ordenação temporal e discriminação de padrões sonoros ${ }^{20}$.

Os critérios de referência para normalidade das habilidades auditivas avaliadas nos testes utilizados foram ${ }^{19,20}$ :

- Teste de Fala com ruído (relação sinal-ruído +5 $\mathrm{dB}$ ) - acertos superiores a $70 \% \mathrm{em}$ ambas as orelhas;

- SSW - para a condição direita competitiva (DC): 7 anos (75\% de acertos); 8 anos ( $80 \%$ de acertos) e 9 anos em diante ( $90 \%$ de acertos). Para a condição esquerda competitiva (EC): 7 anos (65\% de acertos); 8 anos (75\% de acertos) e 9 anos em diante (90\% de acertos);

- Dicótico de Dígitos (Integração binaural) - 7 a 8 anos (orelha direita $-85 \%$ de acertos; orelha esquerda $-82 \%$ ); 9 anos ou mais ( $95 \%$ de acertos em ambas as orelhas);

- TPF versão infantil - 7 a 8 anos (76\% de acertos); 8 a 10 (91\% de acertos);

- TPF versão adulta - 9 a 10 anos (91\% de acertos); acima de 11 anos (96\% de acertos).

Após a primeira etapa de avaliação, os pacientes passaram a ser atendidos durante dez semanas, em sessões semanais individuais de aproximadamente 50 minutos, em salas de terapia comuns. Todas as sessões foram previamente planejadas, de acordo com as habilidades auditivas alteradas na avaliação do processamento auditivo. O programa será mais bem detalhado a seguir.

No final das dez sessões de atendimento fonoaudiológico, todos os pacientes foram reavaliados por meio dos mesmos testes feitos no início do processo.

\section{Descrição do Programa \\ de Treinamento Auditivo}

O programa utilizado ${ }^{21}$ foi organizado com base em estudos de diversos autores, incluindo algumas técnicas inicialmente utilizadas para treinamento auditivo de pacientes surdos e outras desenvolvidas para transtornos do processamento auditivo, bem como orientações para melhora do ambiente acústico e de estratégias de comunicação 8,22-25.

Basicamente, o primeiro passo do programa requer que as habilidades alteradas sejam hierar- 
quizadas, de acordo com o padrão do desenvolvimento auditivo normal, ou seja: detecção, sensação, discriminação, localização, reconhecimento, compreensão e memória. Dentro de cada uma destas etapas, são trabalhadas habilidades auditivas específicas. Assim, hierarquicamente, são trabalhadas as seguintes habilidades auditivas, dentro de cada etapa auditiva:

- Detecção: atenção auditiva a um som ou silêncio (atenção focalizada).

- Sensação / Discriminação: características acústicas do som (espectro, amplitude e tempo); detecção de diferenças entre padrões de estímulos sonoros, fazendo com que o sistema auditivo seja capaz de responder às diferenças entre os diferentes sons; análise e síntese auditivas; fechamento auditivo; consciência fonológica; prosódia.

- Localização: discriminação da direção da fonte sonora e interação binaural.

- Reconhecimento: discriminação e reconhecimento de padrões sonoros (freqüência, intensidade e duração).

- Compreensão: reconhecimento e compreensão de sons na presença de ruído ou mensagem competitiva (atenção seletiva - prestar atenção em um estímulo na presença de outros competitivos; figura-fundo - identificar uma mensagem primária, na presença de sons competitivos).

- Integração: integração de informações auditivas com outros canais sensoriais, bem como integração inter-hemisférica (por exemplo, associação auditivo-visual - associação som-símbolo). São trabalhadas também habilidades como integração / separação binaural.

- Memória: além da habilidade de estocar e recuperar estímulos envolve a habilidade para lembrar a duração ou o número de estímulos auditivos e a memória sequencial (discriminação de sons / padrões sonoros em sequência - ordenação temporal).

Deve-se ressaltar que a atenção (focalizada e seletiva), assim como a memória, permeia todas as outras habilidades auditivas e, portanto, devem ser constantemente trabalhadas, paralelamente às outras habilidades auditivas.

O segundo ponto, fundamental para este programa, é que devem ser trabalhados, na maioria das etapas, sons verbais e não-verbais, com o objetivo de aprimorar mecanismos fisiológicos que, posteriormente, delineiam processos mais complexos do processamento auditivo, bem como estimular ambos os hemisférios corticais, o que exigirá, por várias vezes, transferência inter-hemisférica, que é essencial para diversas habilidades auditivas como integração e separação binaural.
O terceiro passo é respeitar a complexidade de tarefas dentro de cada habilidade auditiva, ou seja, iniciar com atividades menos complexas e, à medida que os acertos forem se tornando mais consistentes, as atividades devem tornar-se mais difíceis. O critério fixado para a mudança de complexidade é que os acertos atinjam $70 \%$ ou mais, não permitindo que os acertos sejam inferiores a $30 \%$, o que indica que as atividades estão muito difíceis e que se deve voltar um passo 4,8,9. Da mesma forma, em relação à hierarquia das habilidades auditivas, deve-se respeitar o critério dos $30-70 \%$, isto é, passar de uma habilidade para outra depois que este alvo seja atingido. Para que isto seja cumprido, todas as atividades realizadas devem ser registradas e os acertos pontuados. Além disso, o fator motivacional é fundamental, ou seja, o paciente deve sempre ser incentivado e seus acertos devem ser ressaltados.

Cabe enfatizar que a complexidade das tarefas pode ser manipulada também por meio da introdução de sons competitivos (fala, música ou ruído) durante a execução de uma determinada atividade, o que torna a tarefa mais difícil. O nível de apresentação dos sons competitivos também pode ser manipulado, facilitando ou dificultando as atividades.

Os estímulos utilizados durante o programa são variados, de acordo com a proposta da tarefa. Os sons de fala podem ser produzidos pelo próprio fonoaudiólogo. Os sons não-verbais podem ser produzidos por diversos instrumentos, fontes sonoras ou pelo $C D$ que faz parte do programa. $O C D$ é composto por 18 faixas que podem ser usadas no treino das seguintes habilidades: detecção (de som ou silêncio - gap), discriminação (de frequência e duração), prosódia (combinação de tons a serem imitados) e reconhecimento (de frequência e duração). Além disso, existe uma faixa com ruído branco para ser usada em atividades de escuta competitiva. O nível de apresentação dos estímulos deve ser confortável ao paciente.

O treinamento auditivo não pode ocorrer separado da família e da escola e, por este motivo, orientações sobre como melhorar o ambiente acústico e estratégias para melhorar a comunicação são dadas aos pais e professores. Além disso, atividades relacionadas com o que está sendo feito em terapia são passadas para os pais, de modo que haja um treinamento auditivo diário, somado ao trabalho feito pelo fonoaudiólogo uma vez por semana, o que é fundamental para o sucesso do programa.

Esta pesquisa foi aprovada pelo Comitê de Ética da Universidade Guarulhos (SISNEP/271 - Parecer

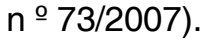

A análise estatística foi feita pelo teste ANOVA. O nível de significância assumido foi 0,05. 


\section{RESULTADOS}

A Tabela 1 ilustra as médias dos resultados obtidos pelos 10 participantes do estudo, antes e depois do treinamento auditivo, para cada um dos testes realizados na avaliação do processamento auditivo. Nota-se que, para ambas as orelhas e para todos os testes, houve melhora das porcentagens de acertos quando comparadas às situações pré e pós-treinamento auditivo. Cabe enfatizar que, com exceção de uma comparação (SSW DC pré e pós-treinamento), a qual apresentou tendência à diferença significante $(p=0,06)$, todas as outras comparações mostraram diferenças estatisticamente significantes, com porcentagens de acertos melhores na situação pós-treinamento.

A seguir, as Figuras 1 a 4 representam as porcentagens de acertos pré e pós-treinamento auditivo para cada participante, em cada um dos testes. Observando-se cada participante, pode-se notar que a maioria teve um aumento das porcentagens de acertos, em ambas as orelhas, na condição pós-treinamento. É importante ressaltar que a performance dos 10 indivíduos, em todos os testes e nas condições pré e pós-treino, foi bastante heterogênea, tanto no que se refere à quantidade de acertos em cada um dos testes e em cada orelha, como no que diz respeito à melhora pós-treino, que foi mais acentuada para alguns indivíduos do que para outros e, às vezes até, mais acentuada em uma orelha em relação à outra.

Com base nas Figuras acima, pode-se notar também que nem todos os participantes atingiram a performance esperada para a idade nos testes realizados, nas situações pré e pós-treinamento (de acordo com os critérios expostos no item Métodos).

Tabela 1 - Média, desvio-padrão (DP) e p-valor referentes às porcentagens de acertos pré e póstreinamento auditivo para cada teste da avaliação de processamento auditivo

\begin{tabular}{|c|c|c|c|c|}
\hline & FR OD pré & FR OD pós & FR OE pré & FR OE pós \\
\hline Média & 68 & 86,4 & 68,4 & 86,4 \\
\hline $\mathrm{DP}$ & 11,31 & 7,35 & 7,64 & 12,53 \\
\hline \multirow[t]{2}{*}{$\mathrm{p}$-valor } & \multicolumn{2}{|c|}{$<0,001^{*}$} & \multicolumn{2}{|c|}{$0,001^{*}$} \\
\hline & DG OD pré & DG OD pós & DG OE pré & DG OE pós \\
\hline Média & 82,75 & 93,05 & 78,75 & 90,3 \\
\hline DP & 13,81 & 7,1 & 13,08 & 6,15 \\
\hline \multirow[t]{2}{*}{$\mathrm{p}$-valor } & \multicolumn{2}{|c|}{$0,050^{*}$} & \multicolumn{2}{|c|}{$0,021^{*}$} \\
\hline & SSW DC pré & SSW DC pós & SSW EC pré & SSW EC pós \\
\hline Média & 70 & 81,6 & 66,25 & 80,05 \\
\hline DP & 12,9 & 12,95 & 13,34 & 13,31 \\
\hline \multirow[t]{2}{*}{$\mathrm{p}$-valor } & \multicolumn{2}{|c|}{$0,060 \#$} & \multicolumn{2}{|c|}{$0,032^{*}$} \\
\hline & TPF OD pré & TPF OD pós & TPF OE pré & TPF OE pós \\
\hline Média & 47,76 & 72,8 & 48,18 & 75,32 \\
\hline DP & 29,44 & 22,98 & 21,76 & 19,13 \\
\hline$p$-valor & \multicolumn{2}{|c|}{$0,048^{*}$} & \multicolumn{2}{|c|}{$0,008^{*}$} \\
\hline
\end{tabular}

Legenda: * - diferença estatisticamente significante; \# tendência à diferença estatisticamente significante (teste ANOVA); FR - Fala com ruído; DG - Dicótico de Dígitos; TPF - Teste de padrão de frequência; OD - Orelha direita; OE - Orelha esquerda. 


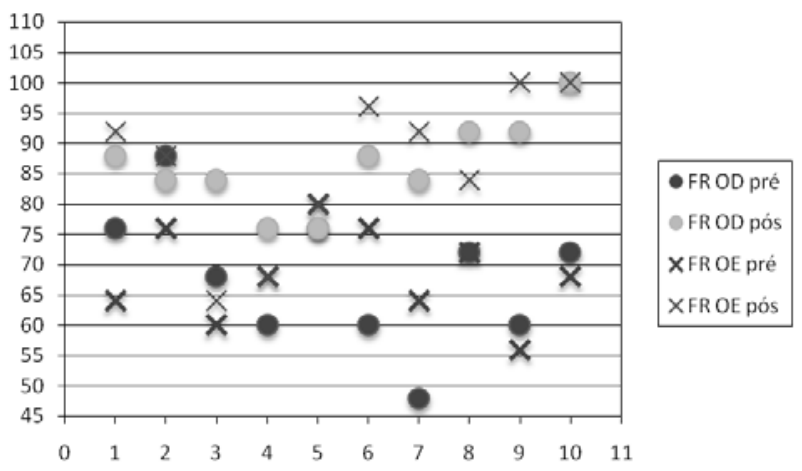

Figura 1 - Porcentagem de acertos pré e póstreinamento auditivo para cada participante (Teste Fala com ruído - FR; OD - orelha direita; OE - orelha esquerda)

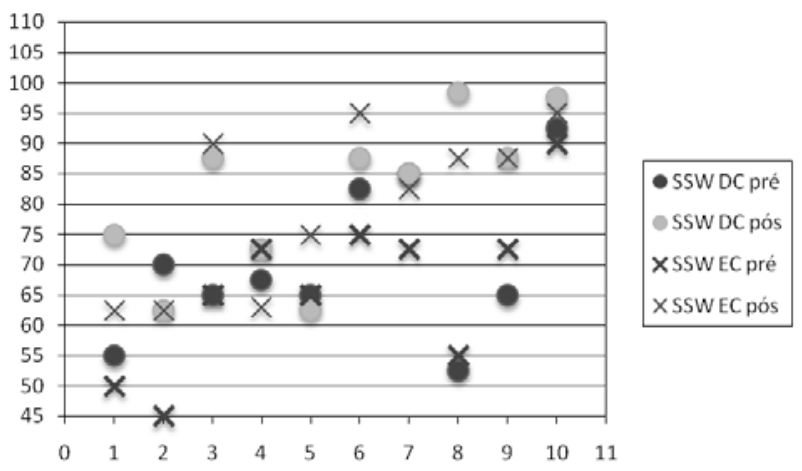

Figura 3 - Porcentagem de acertos pré e póstreinamento auditivo para cada participante (Teste SSW; DC - condição direita competitiva; EC - condição esquerda competitiva)

\section{DISCUSSÃO}

Em casos de transtorno do processamento auditivo, o treinamento auditivo promove uma reorganização neuronal do sistema auditivo e das conexões com outros sistemas sensoriais a ele relacionados, causando uma consequente melhora das habilidades que estavam anteriormente alteradas. Estas mudanças podem ser medidas por meio dos testes auditivos comportamentais ${ }^{2,3,8,9}$, que indiretamente revelam qual a maturidade do sistema auditivo e qual o nível de neuroplasticidade que ocorreu durante o treinamento auditivo.

O presente trabalho buscou demonstrar exatamente isto, ou seja, qual a mudança comportamental promovida por treinamento auditivo específico para transtorno do processamento auditivo em um grupo heterogêneo de pacientes com este tipo de alteração.

Desta forma, com base nos resultados anteriormente relatados, observou-se que todos os sujeitos

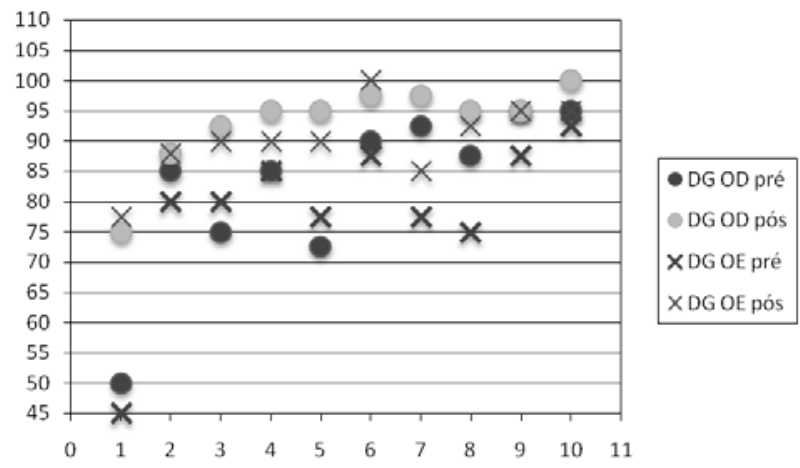

Figura 2 - Porcentagem de acertos pré e póstreinamento auditivo para cada participante (Teste Dicótico de Dígitos - DG; OD - orelha direita; OE - orelha esquerda)

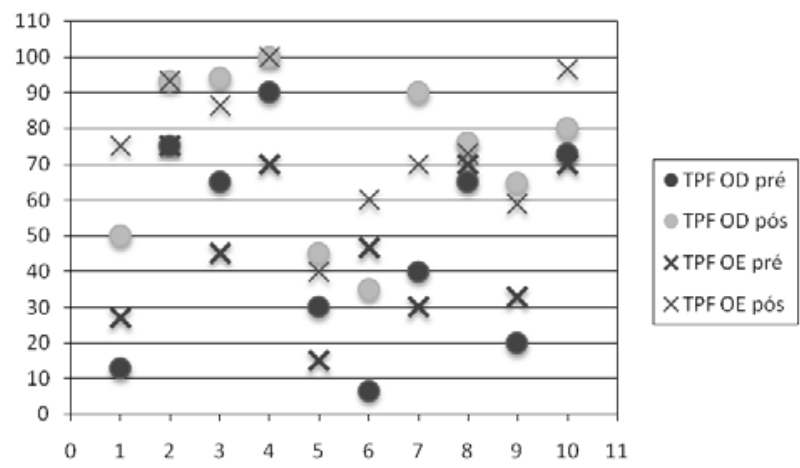

Figura 4 - Porcentagem de acertos pré e póstreinamento auditivo para cada participante (Teste de padrão de frequência - TPF; OD orelha direita; $\mathrm{OE} \mathrm{-} \mathrm{orelha} \mathrm{esquerda)}$

que fizeram parte do estudo tiveram uma melhora significante das habilidades auditivas alteradas. Esta melhora decorrente do treinamento auditivo está de acordo com diversos estudos sobre o assunto, que demonstraram que as habilidades auditivas são passíveis de treinamento e, portanto, de aprendizagem ${ }^{2,7,9,13,15,26-29}$.

De acordo com os resultados, pôde-se notar, também, que o programa de treinamento auditivo não pode ser finalizado ainda com todos os pacientes, uma vez que nem todos alcançaram os critérios de normalidade esperados para sua faixa etária específica. Este fato demonstra que somente 10 sessões não foram suficientes para adequar todas as necessidades de alguns pacientes, que apresentam, além do transtorno do processamento auditivo, outras alterações que dizem respeito à linguagem oral e escrita.

No entanto, o objetivo deste trabalho foi demonstrar a eficácia do treinamento auditivo em pacientes com transtorno do processamento auditivo e 
ele foi alcançado, uma vez que houve diferença estatisticamente significante entre o pré e pósteste, evidenciando que todos os indivíduos tiveram melhora significativa das habilidades auditivas trabalhadas, como também verificado em outros estudos $2,7,9,13,26,27,29$.

A importância do treinamento auditivo nos transtornos do processamento auditivo não pode ser negada e uma intervenção rápida nestas alterações é necessária, como forma de minimizar os prejuízos comunicativos, escolares e sociais que podem aparecer em decorrência destas alterações ${ }^{1-3}$.

Adicionalmente, é fundamental que a intervenção terapêutica seja monitorada por meio de pré e pós-testes, sejam eles comportamentais ou eletrofisiológicos, o que direcionará a atuação do profissional responsável, bem como poderá servir como estratégia motivacional para o próprio paciente pr,-10,26. $^{4}$.

\section{CONCLUSÃO}

O programa de treinamento auditivo informal mostrou-se eficaz em um grupo de pacientes com transtorno do processamento auditivo, uma vez que determinou diferenças estatisticamente significantes entre o desempenho pré e pós-teste na avaliação do processamento auditivo, indicando melhora das habilidades auditivas alteradas.

\section{ABSTRACT}

Purpose: to check the auditory training efficacy in patients with (central) auditory processing disorder, by comparing pre and post results. Methods: ten male and female subjects, from 7 to 20 -year old, took part in this study. All participants were submitted to audiological and (central) auditory processing evaluations, which included Speech Recognition under in Noise, Staggered Spondaic Word, Dichotic Digits and Frequency Pattern Discrimination tests. Evaluation was carried out after 10 auditory training sessions. Results: statistical differences were verified comparing pre and post results concerning the mean percentage for all tests. Conclusion: the informal auditory training program used showed to be efficient for patients with (central) auditory processing disorder considering the patients significant improvement in all post test results.

KEYWORDS: Auditory Perception; Hearing Disorders; Acoustic Stimulation; Neuronal Plasticity

\section{REFERÊNCIAS}

1. American Speech-Language Hearing Association (ASHA). (Central) Auditory Processing Disorders [Technical Report]. [homepage da internet] 2005. [acesso em: 20 jan 2009] Disponível em: URL:http:// www.asha.org/policy.

2. Musiek FE, Shinn J, Hare C. Plasticity, auditory training, and auditory processing disorders. Semin Hear. 2002; 23(4):263-75.

3. Chermak GD. Neurobiological connections are key to APD. Hear J. 2004; 57(4):58-9.

4. Schochat E. Insights for management of processing disorders. Hear J. 2004; 57(10):58.

5. Ohl FW, Scheich $\mathrm{H}$. Learning-induced plasticity in animal and human auditory cortex. Curr Opin Neurobiol. 2005; 15(4):470-7.

6. Mahncke HW, Connor BB, Appelman J, Ahsanuddin ON, Hardy JL, Wood RA, et al. Memory enhancement in healthy older adults using a brain plasticity-based training program: a randomized, controlled study. Proc Natl Acad Sci USA. 2006; 103(33):12523-8.

7. Tallal $P$, Gaab N. Dynamic auditory processing, musical experience and language development. Trends Neurosci. 2006; 29(7):382-90.

8. Chermak GD, Musiek FE. Management: auditory training and metalinguistic and metacognitive strategies. In: Chermak GD, organizador. Central auditory processing disorders: new perspectives. San Diego, CA: Singular Publishing Group Inc.; 1997. p. 185-233.

9. Musiek F, Schochat E. Auditory training and central auditory processing disorders: a case study. Semin Hear. 1998; 19(4):357-66.

10. Jirsa RE. Clinical efficacy of electrophysiologic measures in APD management programs. Semin Hear. 2002; 23(4):349-56.

11. Sweetow RR. Training the adult brain to listen. Hear J. 2005; 58(6):10-7.

12. Johnson KL, Nicol TG, Kraus N. Brain stem response to speech: a biological marker of auditory processing. Ear Hear. 2005; 26(5):424-34. 
13. Moore DR, Rosenberg JF, Coleman JS. Discrimination training of phonemic contrasts enhances phonological processing in mainstream school children. Brain Lang. 2005; 94(1):72-85.

14. Santos JN, Couto ICP, Amorim RMC. Treinamento auditivo em oficinas: opção terapêutica grupal. Pró-Fono. 2006; 18(3):293-302.

15. Kacelnik O, Nodal FR, Parsons $\mathrm{CH}$, King AJ. Training-induced plasticity of auditory localization in adult mammals. PLoS Biol. 2006; 4(4):e71.

16. Musiek F. Auditory training and CAPD: a short history. Hear J. 2006; 59(8):52.

17. Zalcman TE, Schochat E. A eficácia do treinamento auditivo formal em indivíduos com transtorno de processamento auditivo. Rev Soc Bras Fonoaudiol. 2007; 12(4):310-4.

18. Zhou X, Merzenich MM. Developmentally degraded cortical temporal processing restored by training. Nat Neurosci. 2009; 12(1):26-8.

19. Pereira LD, Schochat E. Processamento auditivo central: manual de avaliação. São Paulo: Ed. Lovise; 1997.

20. Auditec. Evaluation manual of pitch pattern sequence and duration pattern sequence. Missouri: Auditec of St. Louis; 1997.

21. Samelli AG, Mecca FFDN. Programa de treinamento auditivo específico para alterações do processamento auditivo. Patente Reg. 10 RTD no 2957522; 2004.
22. Azevedo MF, Pereira LD. Terapia para desordem do processamento auditivo central em crianças. In: Pereira LD, Schochat E. Processamento auditivo central: manual de avaliação. São Paulo: Lovise; 1997. p. 69-77.

23. Giolas TG. Reabilitação aural de adultos com deficiência auditiva. In: Katz J. Tratado de audiologia clínica. São Paulo: Manole; 1999. p. 766-82.

24. Katz J, Wilde L. Desordens do processamento auditivo. In: Katz J. Tratado de audiologia clínica. São Paulo: Manole; 1999. p.486-98.

25. Sweetow RW. The need for auditory training. The ASHA Leader. 2008; 13(5):5-6.

26. Kozlowski L, Wiemes GMR, Magni C, Silva ALG. A efetividade do treinamento auditivo na desordem do processamento auditivo central: estudo de caso. Rev Bras Otorrinolaringol. 2004; 70(3):427-32.

27. Russo NM, Nicol TG, Zecker SG, Hayes EA, Kraus N. Auditory training improves neural timing in the human brainstem. Behav Brain Res. 2005; 156(1):95-103.

28. Neves IF, Schochat E. Maturação do processamento auditivo em crianças com e sem dificuldades escolares. Pró-Fono. 2005; 17(3):311-20.

29. Martins NFC, Magalhães Junior HV. Terapia de processamento auditivo no distúrbio de aprendizagem. Rev Bras Prom Saúde. 2006; 19(3):188-93.

RECEBIDO EM: 26/01/2009

ACEITO EM: 12/10/2009

Endereço para correspondência:

Alessandra Giannella Samelli

Rua Cipotânea, 51 Cidade Universitária

São Paulo - SP

CEP: 05360-000

E-mail: alesamelli@usp.br 